\title{
Role of Healthcare Workers and Anesthesiologists during the COVID-19 Pandemic in India: Past, Present and Future
}

On the eve of first anniversary of Corona Virus Disease 2019 (COVID-19), it is time to pause, look back and think how the year has gone by, its impact on the immediate future and how COVID-19 is likely to change our practices in the future.

According to an unpublished report from the Chinese government, the first case of COVID-19 can be traced back to 17th November 2019, in a 55-year old Chinese citizen from the Hubei province. But the first official report of Severe Acute Respiratory Syndrome (SARS) with COVID-19 by the World Health Organisation (WHO) was dated on 8th December 2019. The first case of COVID-19 in India, was reported on 30th January 2020, in a student who travelled from Wuhan province. ${ }^{1}$ The Government of India's initial response was to impose a stringent and complete lockdown with the objective of providing necessary time for boosting healthcare infrastructure, gearing up manpower with necessary protective equipment and training healthcare workers to combat the impending outburst of the disease. ${ }^{2}$ The lockdown also helped in raising awareness, and highlighting the seriousness of the pandemic in society. Over the next few months, the number of COVID-19 cases increased exponentially and currently, India has the second highest number of cases in the world. It appears that the case fatality rate is very low in India, at about $1.47 \%$, compared to many developed countries. However, case fatality rate estimation may not be reliable as the numbers in numerator as well as the denominator may be inaccurate. Possible undiagnosed and underreported deaths may affect the numerator while the denominator, which is total number of infected people, may not be accurate, as many affected individuals were asymptomatic and remained untested (as confirmed in the serological survey studies).

Despite significant increases in healthcare capabilities across the country, with increase in bed strength, oxygen therapy and Intensive Care Unit (ICU) beds, a shortage in facilities was widely witnessed during the peak of the pandemic. ${ }^{3}$ The current decreasing trend in the COVID-19 wave should not be observed as relief or time to relax. Considering the pandemic scenario in United States of America (USA), United Kingdom (UK) and many European countries, the possibility of second and third wave of infection is looming large and appears to be around the corner. Already, few parts of India like Delhi are witnessing the resurgence of the infection with possibly higher virulence. Fatigue effecting healthcare workers as well as general public is likely to have an adverse impact on the outcome during the second and subsequent waves. Promising results about vaccines from various companies seem like a light at the end of the tunnel. While it remains to be seen as to the efficacy and duration of protection from these vaccines, considering the requirement of stringent cold chain requirement, delivery of the vaccine to such a huge population may in itself pose a separate challenge for the Indian healthcare system. It is not unlikely that COVID-19 cases may decrease but may never disappear, and the disease may remain endemic like many others.

Role of anaesthesiologists: Anaesthesiologists due to their unique knowledge of resuscitation, oxygen therapy and ventilator management along with skills in establishing and maintaining airway devices and indwelling catheters have played a major role as COVID19 warriors. Anaesthesiologists have become the backbone of intensive care management of COVID-19 patients throughout the country. Anaesthesiologists' role as Personal Protective Equipment (PPE) clad warriors during the COVID-19 pandemic has been well appreciated by the public and government authorities during the pandemic than at any other time. In addition to bearing the burden of high risk of exposure to COVID-19 virus while managing aerosol generating procedures, anaesthesiologists have also rendered perioperative care to thousands of COVID-19 patients who underwent emergency surgical procedures including obstetric services. Regional anaesthesia gained widespread acceptance in view of reduced risk of aerosol generation. Most places resumed semi-emergent surgical procedures during the peak of the pandemic itself. Whether to start elective operative procedures or wait for the impending next wave of COVID-19 to pass off remains a dilemma.

Innovations: History is witness to the fact that most scientific developments happen during periods of great stress, like world wars. This pandemic being equivalent to a world war in the field of healthcare, has witnessed many innovations which are likely to impact the future. Like all other branches of life, healthcare delivery also had to accept the "tele" revolution. During the periods of lockdown, teleconsultation gained importance. ${ }^{4}$ Even though the concepts of tele-consultation and tele-ICU were present before, they got a greater impetus during the pandemic. ${ }^{5}$ Diagnosis and treatment of many ailments by video calls provided the benefit of avoiding physical contact with doctors and healthcare workers while saving precious time and reducing the need for travel. Improvisation in broadband penetration and availability of digital infrastructure (which are pre-requisites for telemedicine) helped to a great extent. ${ }^{6}$ Closed circuit television (CCTV) and central monitoring consoles enabling remote monitoring of patients were available in the past also, but this pandemic popularised their use. 7 Many state governments established control room centres, where senior intensivists conducted "tele rounds" and helped in managing ICU patients in peripheral places. At the beginning of the pandemic, most doctors had little experience in management of COVID-19 patients or use of treatment modalities like plasma therapy, remdesivir, tocilizumab, thrombolysis, etc. Tele ICU rounds helped to train doctors in their use and ensured the expertise of intensive care specialists was available even to peripheral facilities. ${ }^{8}$ This has potential to vastly increase the scope and outreach of healthcare delivery in future as well. Tele ICU systems can be used for providing emergency round the clock medical care in remote locations and in ensuring optimal utilization of manpower and resources. Telemedicine and Tele ICU systems hold immense potential in India, as they can mitigate the imbalance of doctor/population ratio in rural and urban areas. Since most of the time, relatives were not present with patients during COVID-19, counselling was mostly conducted through video-conference calls. Even consent for various procedures was taken by audio/video calls, which were recorded and stored. The Government of India aided the wider acceptance of telemedicine by modifying existing legislature which had earlier prohibited prescription of medicines without the patient being physically examined by a doctor. Still many changes in the legal frame work may be necessary, especially with respect to acceptance of consent. Most of the verbal, audio/video consents may not be admissible in the current legal frame work. 
In order to avoid direct contact with contagious patients, many barrier methods were instituted. Importance of hand hygiene in preventing nosocomial and ICU infections has been well documented. But it was always very difficult to train and implement the same. The behavioural change brought about by COVID-19 among healthcare workers hopefully stays in their subconscious memory and can help bring down nosocomial infection rates in ICU in the post-covid era as well.

Before the pandemic, phrases such as "Personal Protective Equipment (PPE)", "N95 mask" were unheard of even in most hospitals and were exclusive to research laboratories. ${ }^{9}$ Local mass production of PPE not only made them available easily, but also brought down their cost significantly. ${ }^{10}$ Proper training in use of PPE with appropriate donning/doffing improved..$^{11}$ Health infrastructure saw tremendous change during a short period. Even high flow nasal oxygen, non-invasive ventilators, video-laryngoscopes etc which were rarely available in many centres were made available. Inability to auscultate with a stethoscope while wearing PPE has increased the use of technologies like ultrasound, chest X-ray and echocardiogram. Digital record keeping rather than paper documentation to reduce the number of fomites is another change which is likely to continue into the post-covid era. Many hospitals successfully used robots for delivery of food, medicine and other essential items to reduce nursing manpower. These technological advancements have potential to change the outlook of future nursing care.

Lockdown was a period when venturing out of home itself was difficult and this motivated many healthcare workers towards an inward journey. The pandemic had a significant impact not just on the physical health but also on the psychological wellbeing of healthcare workers. ${ }^{12}$ Mood disorders such as anxiety and depression were commonly faced..$^{13,14}$ Importance of maintaining work-life balance, role of meditation and spirituality in knowing the inner-self was realised by many. Ultimately resilience of human nature is the most important factor which will help us in emerging victorious in this war. Realisation of the importance of inner journey for a larger cause appears to be the most important silver lining in this pandemic cloud.

\section{Sameer N Desai Umesh Goneppanavar}

\section{References}

1. Ghosh, Aritra, Nundy S, and Mallick TK. How India is dealing with COVID-19 pandemic. Sensors International. 2020;1:100021.

2. The Lancet. India under COVID-19 lockdown. Lancet. 2020;395:1315.

3. Pal R, Yadav U. COVID-19 Pandemic in India: Present Scenario and a Steep Climb Ahead. J Prim Care Community Health. 2020;11:2150132720939402.

4. Chellaiyan VG, Nirupama AY, Taneja N. Telemedicine in India: Where do we stand?. J Family Med Prim Care. 2019;8(6):1872-1876.

5. Lahiri D, Mitra S. COVID-19 is accelerating the acceptance of telemedicine in India. J Family Med Prim Care 2020;9:3785-6.

6. Dash SP. The Impact of IoT in Healthcare: Global Technological Change \& the Roadmap to a Networked Architecture in India. J Indian Inst Sci. 2020; 3:1-13.

7. Naik, B. N., Gupta, R., Singh, A., Soni, S. L., and Puri, G. D. Real-Time Smart Patient Monitoring and Assessment Amid COVID-19 Pandemic - an Alternative Approach to Remote Monitoring. Journal of medical systems. 2020;44:131.

8. Ramakrishnan N, Vijayaraghavan BKT, Venkataraman R. Breaking Barriers to Reach Farther: A Call for Urgent Action on Tele-ICU Services. Indian J Crit Care Med 2020;24:393-397.

9. Vaishya RVA. Protect our healthcare workers: rapid response: "the plight of health care workers in India during COVID-19 pandemic. BMJ 2020; 369:m1324.

10. Yaday N. Indiass PPE Industry: How COVID-19 Demonstrated Untapped Local Production Capacity. India Briefing; 2020.

11. Haji JY, Subramaniam A, Kumar P, Ramanathan K, Rajamani A. State of Personal Protective Equipment Practice in Indian Intensive Care Units amidst COVID-19 Pandemic: A Nationwide Survey. Indian J Crit Care Med 2020; 24:809-816.

12. Shanafelt T, Ripp J, Trockel M. Understanding and addressing sources of anxiety among health care professionals during the COVID-19 pandemic. JAMA. 2020;323:2133-2134.

13. Roy D, Tripathy S, Kar SK, Sharma N, Verma SK, Kaushal V. Study of knowledge, attitude, anxiety \& perceived mental healthcare need in Indian population during COVID-19 pandemic. Asian J Psychiatr. 2020;51:102083.

14. Murthy PR, Venkatesha Gupta KV, Ajith Kumar AK. Is Anxiety a Rising Concern during COVID-19 Pandemic among Healthcare Workers?. Indian J Crit Care Med. 2020;24:369-370. 